Revue des patrimoines

25 | 2014

Le patrimoine de la Grande Guerre

\title{
L'incroyable destin des collections du musée Alfred-Danicourt de Péronne
}

David de Sousa

\section{(2) OpenEdition}

1 Journals

\section{Electronic version}

URL: http://journals.openedition.org/insitu/11479

DOI: 10.4000/insitu.11479

ISSN: 1630-7305

\section{Publisher}

Ministère de la Culture

\section{Electronic reference}

David de Sousa, "L'incroyable destin des collections du musée Alfred-Danicourt de Péronne », In Situ [Online], 25 | 2014, Online since 10 December 2014, connection on 25 June 2020. URL : http:// journals.openedition.org/insitu/11479; DOI : https://doi.org/10.4000/insitu.11479

This text was automatically generated on 25 June 2020 .

\section{(c) (i) (9)}

In Situ Revues des patrimoines est mis à disposition selon les termes de la licence Creative Commons Attribution - Pas d'Utilisation Commerciale - Pas de Modification 4.0 International. 


\title{
L'incroyable destin des collections du musée Alfred-Danicourt de Péronne
}

\author{
David de Sousa
}

\section{Un musée rêvé par un maire et un sous-préfet}

1 «Il est bien regrettable que vous habitiez aujourd'hui aussi loin de nous et que vous ne soyez plus là pour stimuler d'une façon intelligente le zèle de la jeune génération ${ }^{1} »$ : c'est en des termes empreints de respect et d'émotion que le maire de Péronne Charles Alfred Danicourt (1837-1887) s'adresse dans une lettre de 1881 à Georges Vallois (1827-1892), ancien sous-préfet d'arrondissement. Les deux hommes ont fait connaissance plus de vingt ans auparavant, en 1859, lorsque Vallois prend son poste à Péronne après quelques années à la tête de la sous-préfecture de Sélestat. Licencié en droit, le représentant de l'État est un amateur éclairé d'histoire et d'archéologie. Notable de province et collectionneur comme son père avant lui, Danicourt est quant à lui passionné de beaux objets et de littérature ${ }^{2}$ : leur rencontre, puis leur amitié, aboutiront à la création de l'un des plus beaux musées de Picardie au XIX siècle.

2 En poste durant onze années (!) à Péronne où il fonde une famille, le haut fonctionnaire passe son temps libre à revisiter l'histoire locale, identifier de nouveaux sites archéologiques, et tente inlassablement de transmettre sa passion pour la culture. Membre de plusieurs sociétés savantes, Vallois publie quelques ouvrages et articles de vulgarisation dont la qualité de la démarche historique est remarquable pour l'époque. Surtout, il trouve en Alfred Danicourt un "élève » avide de connaissances archéologiques et de rigueur scientifique. Il le fait entrer à la Société des antiquaires de Picardie, le guide dans ses premières études d'artéfacts. Les deux hommes se consultent, se conseillent, s'échangent leurs publications, s'en signalent de nouvelles. Ils confrontent leurs découvertes et leurs théories sur les vestiges locaux. Nommé secrétaire général de la préfecture du Cher, Georges Vallois quitte Péronne en 1870 non 
sans avoir profondément semé dans l'esprit d'Alfred Danicourt les germes d'une future institution muséale péronnaise, et même d'une société savante locale.

Fils d'avoué et petit-fils de notaire, Alfred Danicourt gère une partie du patrimoine de quelques-unes des grandes familles du secteur de Péronne. Il s'investit parallèlement dans ses mandats de conseiller municipal puis de maire. Il se bat pour le relèvement de Péronne après les destructions du siège de 1870-1871 par les Prussiens, et pour la restauration de l'église Saint-Jean-Baptiste bombardée. Convaincu par son ami souspréfet de la nécessité d'établir un musée à Péronne, le maire lance le projet en $1874^{3}$. Durant les trois années qui suivent, Alfred Danicourt accumule les objets inédits, obtient des dons privés et des dépôts de l'État. C'est un musée déjà richement doté qu'il inaugure le 7 octobre 1877 après l'avoir fait aménager dans la mairie, sur ses propres deniers en complément d'une souscription publique. Danicourt a voulu ce lieu comme un espace de découverte de l'archéologie du monde tout autant qu'un lieu de conservation et de promotion de l'histoire et de l'art de la Somme. Il se porte souvent acquéreur des trouvailles anciennes faites dans sa région, et s'appuie sur plusieurs antiquaires qui lui signalent les plus belles pièces sur le marché national. C'est comme cela qu'il acquiert par exemple à Bavay ${ }^{4}$, en 1881, trois fibules gallo-romaines zoomorphes et deux petits bronzes d'Apollon et Mercure lors de la vente de la collection Crapez ${ }^{5}$. C'est aussi à travers le monde que Danicourt poursuit sa quête de raretés : il se rend partout en Europe, en Afrique du Nord, et jusque sur les bords du Nil. De chacun de ses voyages il ramène en Picardie bijoux en or et objets précieux ${ }^{6}$.

4 Le 5 août 1887, lors de la lecture du testament d'Alfred Danicourt, le conseil municipal apprend que le musée de Péronne est destinataire de toutes les collections, et la bibliothèque municipale de tous les ouvrages originaux que l'ancien maire a réunis dans sa demeure péronnaise ou dans son appartement parisien ${ }^{7}$. Au 16 de la place Vendôme, les émissaires de la Ville prennent possession d'une vitrine contenant la plus grande fierté de Danicourt: son exceptionnel médaillier gaulois, composé de plus de 500 monnaies dont un rarissime statère d'or de Vercingétorix ${ }^{8}$. De plus, la Ville reçoit 232000 francs pour financer notamment la pérennisation des activités du musée et son agrandissement. C'est pourquoi le jour des funérailles de l'évergète, le sous-préfet d'arrondissement ne parle plus dans son discours du «musée de Péronne » mais bien du « musée Danicourt »" 
Figure 1

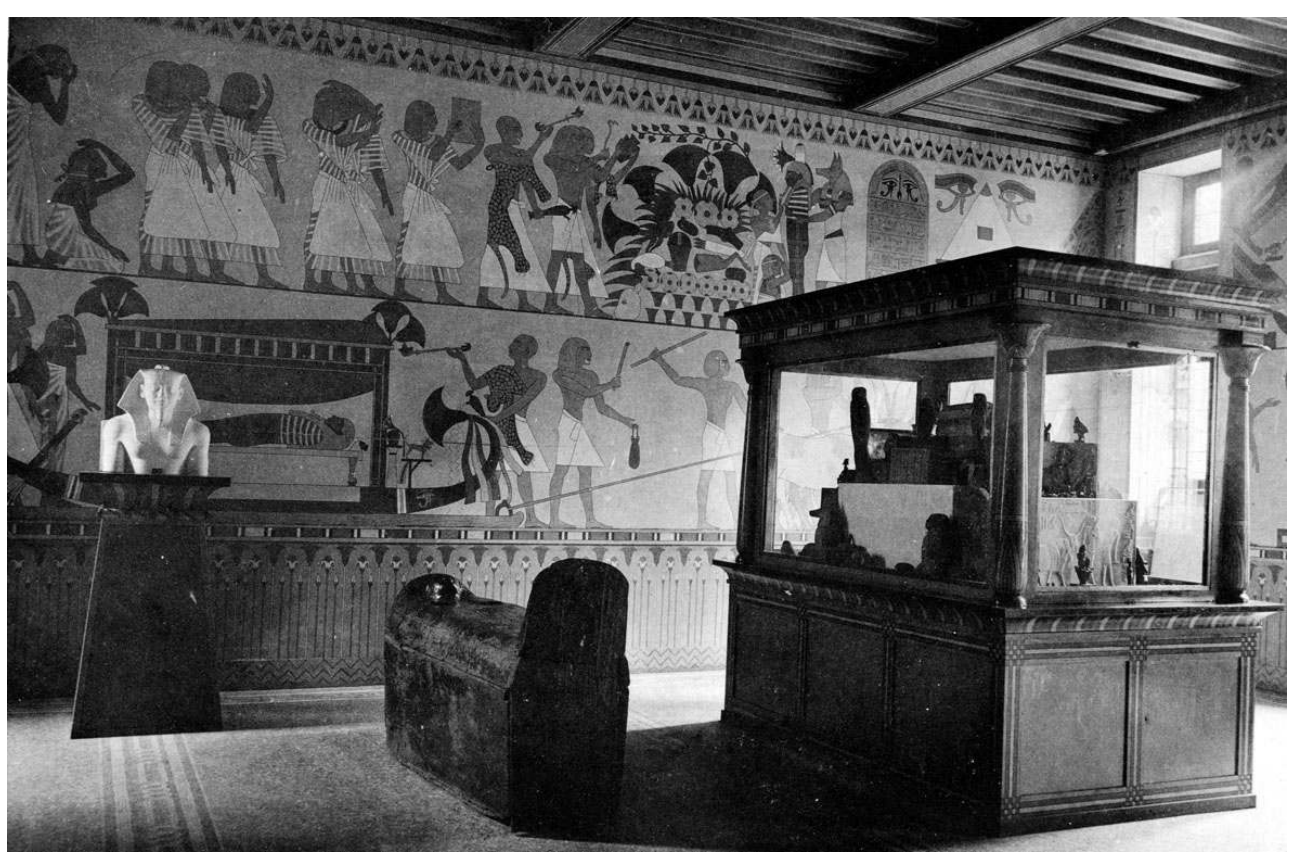

La salle d'égyptologie du musée vers 1906.

Phot. Souillard, E., coll. Musée Alfred-Danicourt. @ Musée Alfred-Danicourt.

5 Dans la décennie qui suit, le musée poursuit son enrichissement jusqu'à occuper sur deux niveaux toute l'aile gauche de l'hôtel de ville. Les antiquités égyptiennes ${ }^{10}$ (fig. 1) y côtoient la numismatique et l'orfèvrerie antiques, l'archéologie médiévale, la production artistique picarde, ou encore les souvenirs des guerres passées (fig. 2). La mémoire historique de Péronne et de tout l'est de la Somme y est particulièrement conservée. Les collections croissent si rapidement que Félix Louis, conservateur à partir de $1898^{11}$, se voit contraint d'installer des tableaux dans le cabinet du maire, la salle $d u$ conseil ou la salle des fêtes. La commission municipale musée-bibliothèque finit d'ailleurs par refuser les dons, faute de place $^{12}:$ le musée de Péronne est l'un des phares culturels de Picardie à la veille de la Première Guerre mondiale. 
Figure 2

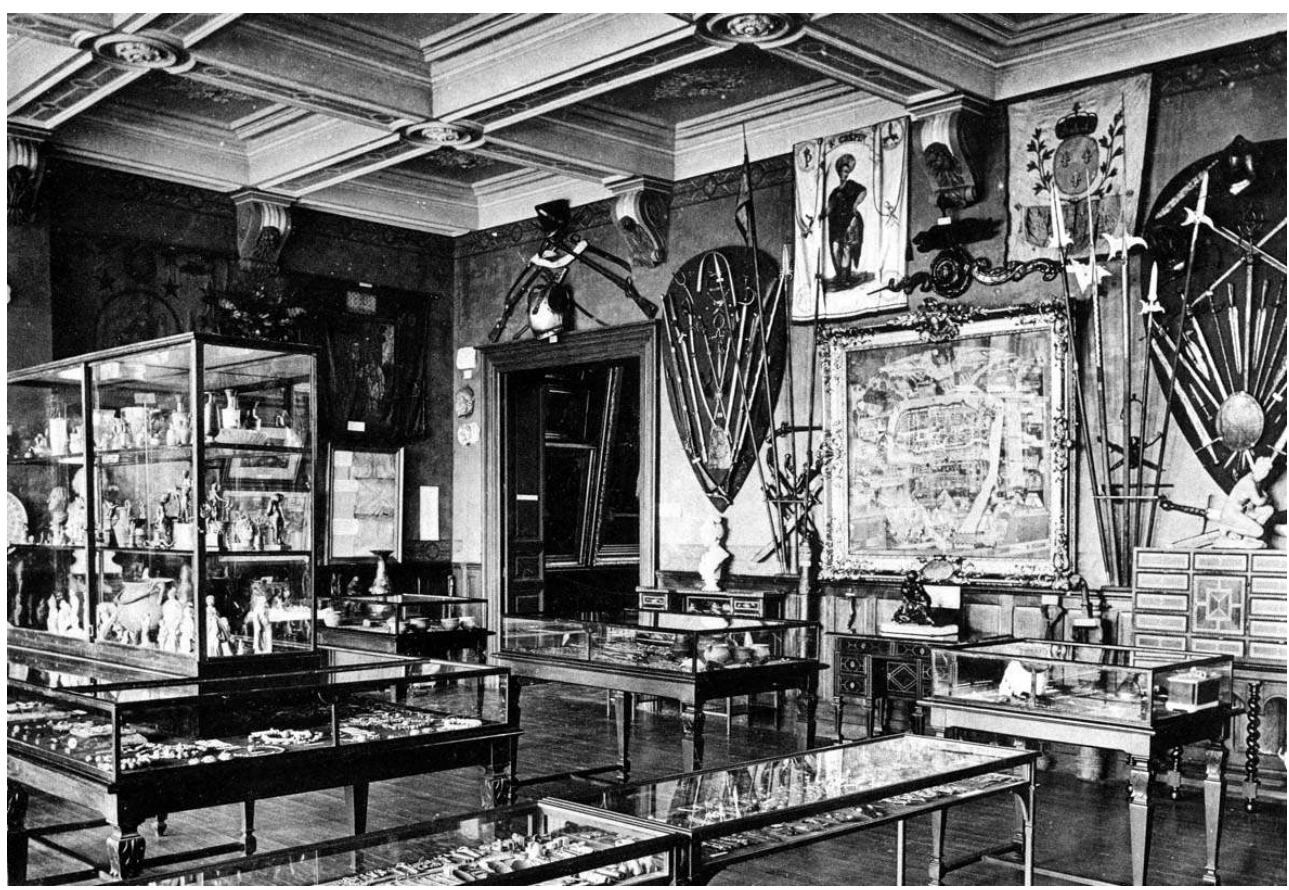

L'une des salles d'archéologie du musée vers 1906.

Phot. Souillard, E., coll. Musée Alfred-Danicourt. () Musée Alfred-Danicourt.

\section{Un musée victime de guerre}

La ville de Péronne est solidement investie par les troupes allemandes à partir du 24 septembre 1914 : elles la conservent jusqu'en mars 1917. L'hôtel de ville est choisi comme centre de commandement durant ces deux ans et demi d'occupation. Alors que l'horloge du campanile de la mairie est mise à l'heure d'outre-Rhin, les officiers s'installent confortablement dans les salles de l'étage et du musée; la galerie d'égyptologie du rez-de-chaussée sert quant à elle de corps de garde. Quotidiennement, une commission municipale, hâtivement formée après la fuite du maire et des principaux fonctionnaires, agit depuis l'une des salles du bâtiment. Elle fait le lien entre la Kommandantur, les états-majors successifs, et la population. Garante de l'interdiction officielle des pillages, elle conserve un œil attentif sur toutes les activités des troupes allemandes et sur les biens de la Ville. Ainsi, lorsque des tableaux et des panoplies d'armes anciennes sont empruntés en novembre 1915 pour décorer une villa occupée par le duc de Saxe-Meningen en visite sur le front, ils sont ensuite replacés au musée. Cette garde vigilante des Péronnais permet que les collections du musée AlfredDanicourt soient encore intactes à la veille du déclenchement de la bataille de la Somme.

7 Un déluge de feu s'abat sur Péronne à partir du 24 juin 1916. L'offensive du $1^{\text {er }}$ juillet porte un premier coup très dur au musée : un obus traverse le toit et explose au sein des salles du premier étage. L'autorité municipale reçoit le samedi 8 juillet l'ordre de préparer l'évacuation de tous les civils. Ces derniers font face à des soldats allemands isolés qui forcent les portes des maisons à coups de hache, ravagent les magasins, pillent les stocks. Les derniers habitants sont emmenés de force le 22 juillet 1916 : 
pendant près de huit mois, les soldats allemands restent les seuls maîtres de la ville. Les caves sous la mairie ${ }^{13}$ sont transformées en hôpital. On y descend des tableaux du musée, du mobilier pour les officiers, et même un piano. En surface, tout est ravagé. Hugo Natt, médecin chef du $118^{\mathrm{e}}$ régiment d'infanterie de l'armée allemande témoigne en février 1917 :

Si on entre par la place du marché et au-dessus des tas de gravats à l'intérieur de l'hôtel de ville, on arrive dans une grande salle [...]. On dit qu'ici il y a eu une collection importante de monnaies. Dans les ordures et la saleté qui couvrent le sol, se trouvent des fragments de reproductions en plâtre, des parties de squelettes, et pas mal d'objets du musée [...]. C'est particulièrement apocalyptique ${ }^{14}$ (fig. 3).

Figure 3

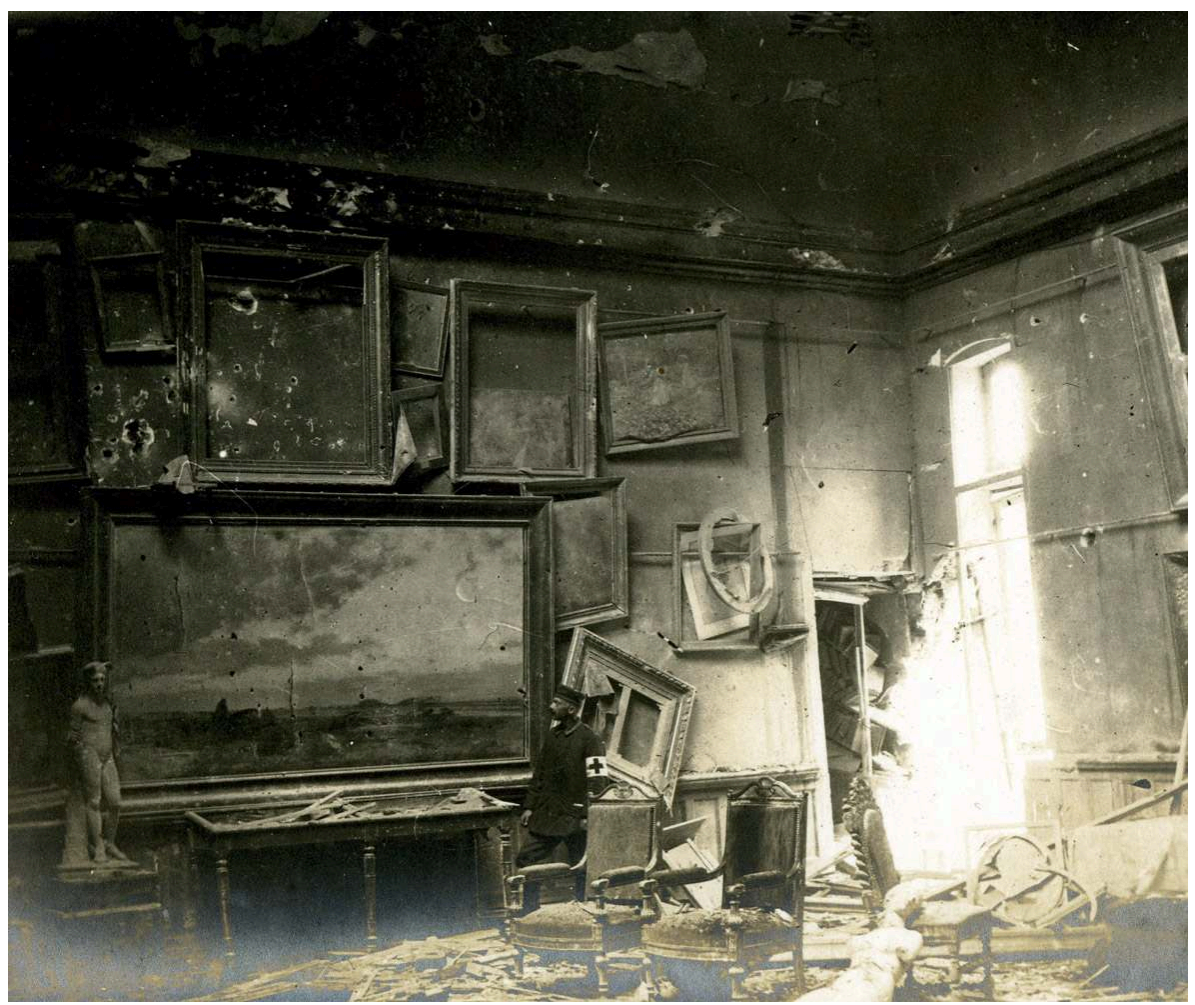

La grande salle des peintures du musée vers la fin 1916.

Coll. Musée Alfred-Danicourt. @ Musée Alfred-Danicourt.

Le 18 mars 1917, les Britanniques pénètrent dans Péronne après la retraite des troupes allemandes sur la ligne Hindenburg. S'avançant au milieu de ruines abandonnées et souvent encore fumantes, ils découvrent sur l'hôtel de ville défiguré, là où se trouvait l'étage du musée, une énorme pancarte (fig. 4) sur laquelle on peut lire l'inscription Nicht ärgern, Nur wundern!, ce qui peut se traduire par « Ne pas se fâcher, seulement s'étonner ! ${ }^{15} »$. Les milliers d'objets qui composaient les collections péronnaises semblent n'avoir pu échapper au désastre. Le conseil municipal réuni en novembre 1917 confirme que des richesses du musée, on n'a retrouvé in situ qu'un buste en marbre, un portrait et une table. Le maire Charles Boulanger précisera en 1923 que « [...] l'enlèvement des collections par les Allemands a été systématique ; ce qui en donne bien la preuve, c'est que lorsqu'on a déblayé les salles du musée, aucun vestige n'a été trouvé parmi les décombres ${ }^{16} »$. 
Figure 4

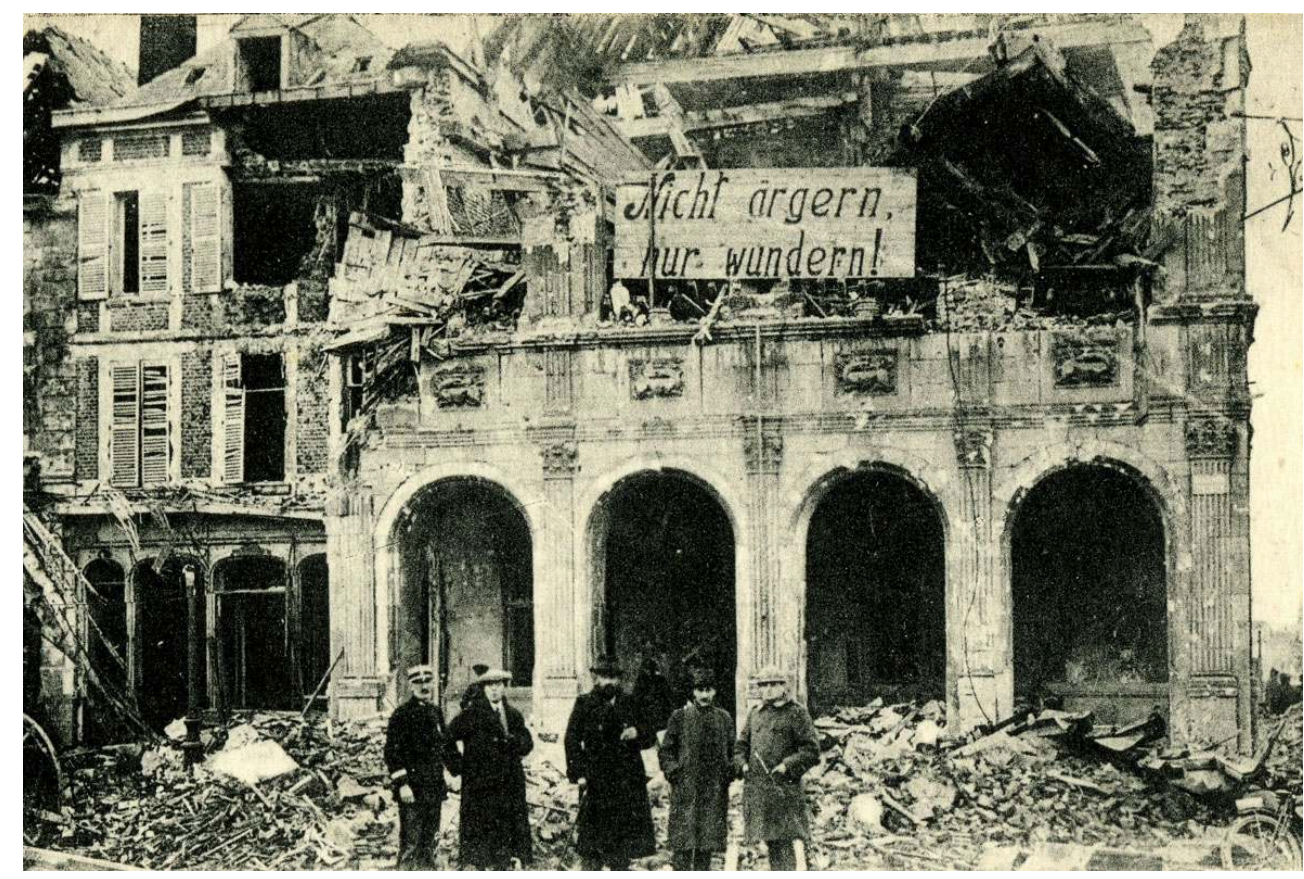

La mairie et l'étage du musée en ruines en avril 1917. Carte postale ancienne.

Coll. Musée Alfred-Danicourt. @ Musée Alfred-Danicourt.

Pourtant, une petite partie des collections a tout de même échappé à la destruction ou au pillage, le conseil municipal adresse en effet « [...] des remerciements très vifs votés à $\mathrm{M}$. Louis, conservateur du musée, pour avoir sauvé des griffes des Allemands une quantité importante d'objets rares et précieux du musée ${ }^{17}$.» En effet, c'est quelques jours avant l'entrée des Allemands dans Péronne fin août 1914, et son propre départ de la ville, que Félix Louis, assisté du gardien de police Anatole Gronier ${ }^{18}$, prit la décision de cacher une partie des trésors du musée sous un étau dans le sol d'un atelier de serrurerie ${ }^{19}$. Une petite caisse scellée fut ainsi enterrée, renfermant près d'un millier de monnaies précieuses, de bijoux antiques, un ensemble de très beaux objets mérovingiens, et quelques bronzes et terres cuites. Limité dans ses choix par les dimensions du contenant, nous pouvons penser que Louis privilégia les éléments les plus rares et précieux par rapport à ceux d'histoire locale auxquels tous étaient pourtant si attachés. Le registre d'inventaire est lui aussi porté disparu; ne restent que des éléments lacunaires. Mais il suffit de regarder les photographies des salles du musée d'avant 1914, les vitrines remplies d'objets et les murs chargés d'œuvres, pour prendre la mesure de tout ce que Péronne a perdu, soit environ $95 \%$ des collections permanentes et la totalité des dépôts de l'État. Si certaines œuvres purent être éloignées $d u$ front par l'autorité militaire allemande pour leur protection ${ }^{20}$, il est certain qu'un «pillage troupier » acheva le ravage des salles du musée entre 1916 et 1918.

10 C'est dans les années 1930 que l'on apprend comment Félix Louis revint à Péronne en avril 1917 grâce à un sauf-conduit ${ }^{21}$. Il se rendit discrètement dans l'atelier complètement détruit, creusa, et retira du sol la petite caisse intacte. La ville restant alors sous autorité militaire anglaise, il décida de placer sa charge sous la protection des autorités françaises à Amiens. Son retour, caisse sur l'épaule, fut des plus épiques. Il monta d'abord clandestinement dans un train pour Amiens. Le lendemain, il se rendit 
successivement à la préfecture puis à la mairie, où personne n'accepta de prendre en charge les objets. La préfecture lui proposa alors de déposer la caisse dans un coffre de banque, mais après un essai il s'aperçut que les dimensions de la caisse le lui interdisaient. C'est finalement le concierge du musée d'Amiens qui, sur ordre de la préfecture, accepta à contrecœur la caisse. Selon le Courrier de Péronne, la caisse aurait ensuite suivi les collections du musée d'Amiens, évacuées en 1918 vers Rouen pour les protéger de l'offensive allemande ${ }^{22}$. Elle serait revenue à Péronne dans les années 1921-1922; ce retour n'est pas documenté.

\section{Des collections reconstituées grâce aux dommages de guerre}

L'organisation du déblaiement des ruines, précédant la mise en place de l'habitat provisoire et la restauration des services publics, ne laisse que peu de temps à la municipalité pour réfléchir au devenir des objets perdus ou sauvés. Les collections disparues ne sont pourtant pas oubliées dans la déclaration de dommages de guerre; le préjudice de la ville pour son musée est estimé par F. Louis à près de 586000 francs. Ce chiffre tient compte de la valeur des quelques objets récupérés via le Séquestre général des biens restitués par les Allemands ${ }^{23}$. Le conservateur reçoit en avril 1921 un cartel Louis XIV, quatre tableaux et deux statuettes, le tout envoyé depuis un dépôt de Maubeuge. Puis, en juillet 1922, c'est depuis un dépôt de Metz qu'on lui renvoie la collection de monnaies romaines ${ }^{24}$. Ces restitutions confortent la municipalité dans l'avis qu'elle s'est forgé d'une totale responsabilité des troupes allemandes dans la disparition des collections du musée Danicourt.

12 L'hôtel de ville, lieu d'exposition des collections depuis leur constitution presque cinquante ans auparavant, devient rapidement la priorité dans le plan de reconstruction. Son relèvement sera le symbole de la restauration définitive de la souveraineté française sur la ville. On peut s'étonner aujourd'hui qu'aucune salle ne soit alors destinée au musée dans le nouvel édifice. Le fait est encore plus étonnant quand on sait que parmi le jury du concours architectural figurait le peintre péronnais Alfred Rey, artiste exposé en bonne place dans l'ancien musée. Le constat du très faible nombre d'objets à présenter fut certainement déterminant. Le petit ensemble pourrait simplement tenir dans quelques vitrines placées, par exemple, dans la nouvelle bibliothèque municipale.

En 1927 se déroule la Fête de la Renaissance, marquant l'inauguration du nouvel hôtel de ville, des écoles, de la gendarmerie et de quelques habitations. La véritable reconstitution des collections du musée commence après le versement des indemnités de dommages de guerre pour ces dernières ${ }^{25}$ et alors qu'aucun nouvel espace d'exposition n'a été prévu pour les accueillir. Plusieurs toiles sont achetées lors d'expositions organisées par l'association artistique péronnaise La Croûte ${ }^{26}$. Parallèlement, la commission municipale musée-bibliothèque décide en juin 1929 de s'adresser directement aux ateliers des artistes. Des accords d'achat sont ainsi passés avec Jules Adler, Maurice Asselin, Charles Atamian, Maurice Cahours, Louis DésiréLucas, André Devambez, Marie Réol ou encore Raymond Tellier. Les ventes aux enchères sont régulièrement fréquentées par quelques délégués municipaux. Ce sont au total une cinquantaine d'œuvres (huiles sur toile, aquarelles, dessins) qui sont achetées 
entre 1927 et 1935 afin de reconstituer la collection beaux-arts ; rien n'est prévu pour étoffer le fonds archéologique.

L'année 1935 marque la fin de cette croissance rapide de la collection artistique : les derniers crédits tirés des indemnités de dommages de guerre sont épuisés en novembre. Mais le volume important de ces achats interdit désormais une réinstallation dans une salle de la Porte de Bretagne ${ }^{27}$, solution un temps envisagée. Quelques objets archéologiques sont exposés, sous la surveillance de Félix Louis, dans la salle de lecture de la bibliothèque; une majorité se trouve en coffre à la Banque de France. Certains tableaux sont accrochés au gré des couloirs du nouvel hôtel de ville ; la plupart sont stockés dans une petite salle de l'édifice transformée en réserve provisoire. Des dons des familles d'artistes ou de notables s'ajoutent à ces achats: la Ville est alors à la tête d'une centaine de toiles et de quelques ensembles archéologiques précieux sans aucun lieu approprié où les offrir au regard du public.

\section{Une nouvelle guerre redonne une place au musée}

15 Alors qu'à la fin des années 1930 une réinstallation du musée dans le château médiéval semble faire consensus, le conservateur se retrouve plongé, par quelques courriers officiels, dans un passé encore très proche. Dans le contexte international tendu et qui laisse présager une nouvelle guerre, le ministère de l'Éducation nationale et des BeauxArts ne souhaite pas livrer les conservateurs provinciaux à eux-mêmes comme ce put être le cas en 1914. Dans plusieurs notes confidentielles, dès septembre 1938, il est demandé au conservateur de Péronne de s'assurer de la disposition de caisses à utiliser pour une éventuelle mise à l'abri des œuvres d'art. Portant des inscriptions codées, ces caisses seraient dirigées par les autorités civiles vers des centres de recueil à définir, d'où elles seraient conduites vers d'autres départements par des officiers de réserve appartenant à la direction des Beaux-Arts ${ }^{28}$.

16 Le 23 août 1939, un télégramme codé convainc le maire d'ordonner la mise en caisse des trésors déjà protégés en 1914. Un peu plus de 1500 objets sont ainsi à nouveau enfermés, dont deux tiers de monnaies. Les tableaux, qu'il est matériellement impossible d'évacuer en totalité, sont pour certains enfermés dans des caisses, puis tous descendus dans les sous-sols de l'hôtel de ville, mis à l'abri des bombardements mais livrés à l'humidité. On sait aujourd'hui que ce fut là une heureuse initiative, car la salle qui leur servait de réserve fut complètement détruite par des bombardiers allemands le 17 mai 1940. Ce jour-là, toute l'aile droite de l'hôtel de ville est soufflée. L'ordre d'évacuation est donné, et Péronne se vide de ses habitants. Le conseil municipal, le personnel communal et le sous-préfet se replient jusqu'à Saint-Lô, dans la Manche. La caisse du musée est finalement du voyage : aucun agent du ministère n'est venu la prendre en charge.

17 La municipalité de Péronne et ses services sont de retour à la fin août 1940, après l'armistice. Félix Louis, très âgé, ne reprend pas ses fonctions de conservateurbibliothécaire : son poste est alors confié à Gustave Devraine ${ }^{29}$. C'est ce dernier qui reçoit la tâche de cacher la caisse du musée de retour, elle aussi, à Péronne : le périple des objets se poursuit en secret jusque dans la chambre à coucher de la concierge de la mairie ${ }^{30}$. C'est pourtant un officier allemand bien renseigné qui se présente à la mairie en mars 1941, et obtient de voir les objets qu'il souhaite faire "dessiner " par des collègues $^{31}$. Pour la seconde fois, les trésors péronnais sont alors soustraits à l'intérêt 
allemand. D'un commun accord, le maire et Devraine décident de les faire disparaître ; le maire refuse de connaître la cachette. Devraine s'adresse donc au directeur des travaux de la Ville: ils descendent tous deux dans un des anciens cachots sous la mairie, puis enterrent la caisse dans le sol en terre battue. Pour plus de sûreté, des ouvriers recouvrent le lendemain le sol du cachot par une dalle de béton.

Lorsque les Allemands reviennent ${ }^{32}$, on leur déclare que la caisse a été volée ${ }^{33}$. Les objets restent à l'abri jusqu'en septembre 1944 et la libération de Péronne. La joie de récupérer les trésors archéologiques ne peut alors occulter la déception de constater la ruine quasi complète des pastels et des aquarelles, et l'altération de certaines peintures à l'huile, victimes de l'humidité des souterrains. Soutenu par la direction des Musées de France $^{34}$, Gustave Devraine convainc la Ville de décider la création de nouvelles salles pour le musée dans l'aile de la mairie à reconstruire ${ }^{35}$. Enfin, le musée Alfred-Danicourt rouvre officiellement ses portes le 3 juillet 1955. En plus d'une exposition temporaire de peinture hollandaise, les visiteurs redécouvrent tous ces trésors archéologiques que l'on n'avait plus exposés ensemble depuis le mois d'août 1914.

Le musée municipal de Péronne fut le seul musée samarien complètement pillé et détruit lors de la Grande Guerre. Gloire de Péronne au XIX siècle, il est oublié par la première Reconstruction et c'est par la seconde qu'il retrouve une place dans l'hôtel de ville. Nous pouvons frissonner en lisant dans ses archives que dans le climat de Guerre froide qui s'installait, le conservateur Gustave Devraine reçut après 1955 une nouvelle circulaire confidentielle sur la protection des œuvres d'art en cas de prochain conflit. Il répondit qu'il savait déjà - presque par tradition - comment protéger les trésors du musée de Péronne, "[...] l'encombrement étant minime et la nature des objets ne présentant aucun risque de détérioration ${ }^{36}$.». C'est aussi par les actes de courage individuels de ses conservateurs que ce musée victime de guerre, toujours blotti au sein de l'hôtel de ville deux fois reconstruit de Péronne, continue de présenter de surprenantes collections archéologiques en numismatique (fig. 5), glyptique, ou orfèvrerie, héritage à travers les guerres du rêve d'un maire et d'un sous-préfet. 
Figure 5

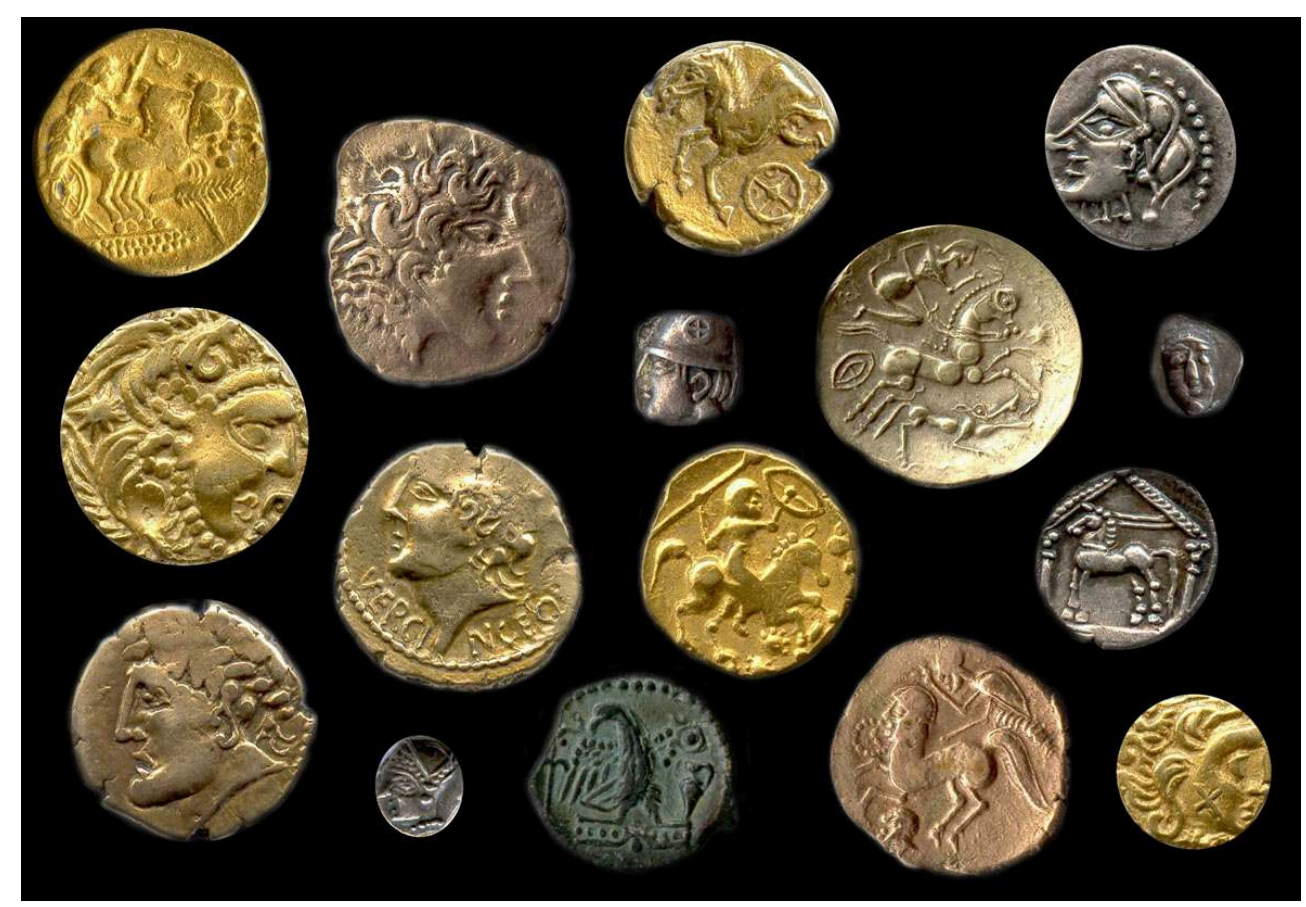

Extrait du médaillier gaulois d'Alfred-Danicourt.

Phot. de Sousa, D., coll. Musée Alfred-Danicourt. @ Musée Alfred-Danicourt.

\section{NOTES}

1. - Archives du musée Alfred-Danicourt, fonds Vallois.

2. - En 1882, Alfred Danicourt rachète une partie de la collection d'intailles du comte de Gobineau. C'est probablement pourquoi dans le Livre des Collectionneurs d'Alphonse Maze-Sencier (1885), Danicourt n'est répertorié que comme « curieux de camées et d'intailles ». L'inventaire de ses collections après son décès ne laisse pourtant aucun doute sur l'éclectisme de ses centres d'intérêt, de la Préhistoire à la peinture académique.

3. - Archives reconstituées de la ville de Péronne, 1874.

4. - Une partie du présent article a fait l'objet d'une publication au sein du catalogue de l'exposition Voyage à travers les collections de Bavay: histoire d'archéologie 1780-1976, Forum antique de Bavay, 2013.

5. - Cet ensemble d'antiquités dites romaines, provenant presque exclusivement de fouilles à Bavay, avait été réuni entre 1820 et 1860 par l'ancien maire Anatole Crapez.

6. - Danicourt rédige plusieurs articles pour la Revue Archéologique, consacrés à la description de certaines de ses acquisitions. Il publie aussi Une révolte à Péronne sous le gouvernement du Maréchal d'Ancre (Péronne ; 1885).

7. - Archives reconstituées de la ville de Péronne, 1887. 
8. - Dans une lettre de 1879 adressée à Georges Vallois, Alfred Danicourt lui avoue que ce statère "[...] lui a coûté le blanc des yeux », soit 11000 francs à l'époque ; archives du musée AlfredDanicourt, fonds Vallois.

9. - L'indépendant de Péronne, 31 juillet 1887. En 1910, une avenue de Péronne prend aussi le nom de Danicourt.

10. - Alfred Danicourt avait fait réaliser en 1878 sur les murs de la salle, par Georges Legrain, élève de l'École du Louvre, des reproductions de peintures tirées de l'étude des tombeaux royaux de l'Égypte ancienne.

11. - Ce poste vient en complément de ses activités en tant que directeur de l'agence péronnaise de la Banque de France. La désignation des conservateurs à Péronne se fait alors conjointement par le maire et le préfet.

12. - Archives du musée Alfred-Danicourt, dons, legs et dépôts, 1908.

13. - Toute l'aile gauche du bâtiment, investie par le musée, est en fait l'ancien bailliage (tribunal) de la ville vendu par le conseil général en 1863 et rattaché à la mairie. À six mètres sous la surface se trouvent encore aujourd'hui de longs couloirs et les anciens cachots du bailliage qui furent des lieux d'abri pour la population ou les combattants pendant les guerres contemporaines.

14. - HIRSCHFELD, G., KRUMEICH, G., RENZ, I. Die Deutschen an der Somme 1914-1918. Krieg, Besatzung, Verbrannte Erde. Essen : Ed. Klartext, 2006.

15. - Intégrée aux collections de l'Imperial War Museum de Londres après la guerre, cette pancarte est toujours visible à Péronne, prêtée et exposée au sein de l'Historial de la Grande Guerre.

16. - Rapport manuscrit du maire de Péronne, phrase soulignée sur le document original, archives départementales de la Somme, 10 R 1032.

17. - Archives municipales de Péronne, registre des délibérations, 1917.

18. - La participation d'Anatole Gronier au sauvetage des collections n'est connue que depuis 2012 et la remise au musée, par un descendant du gardien, de plusieurs documents manuscrits relatant les événements.

19. - Le Courrier de Péronne, 31 août 1930.

20. - Un des portraits "enlevés» du musée de Péronne fut exposé en 1917 dans un musée temporaire établi par les Allemands à Maubeuge. L'œuvre est même publiée dans le catalogue Das Museum Au Pauvre Diable zu Maubeuge, par D. Frh.v. Hadeln, Lt. D. R. Stuttgart : 1917.

21. - Le Courrier de Péronne, série d'articles, septembre 1930.

22. - Selon l'ouvrage d'A. Chatelle, Amiens pendant la guerre 1914-1918, une majeure partie des collections du Musée d'Amiens fut expédiée par camions en 1918 dans les communes d'Eu et Martainville (Seine-Inférieure).

23. - Archives du musée Alfred-Danicourt.

24. - Les monnaies romaines en or répertoriées avant 1914 resteront manquantes. Gustave Krauß, sous-officier allemand, nous dit dans un rapport que le 15 juillet 1916, «[...] L'hôtel de ville commencait à brûler et la collection de monnaies romaines qui s'y trouvait fut emportée par le lieutenant Hofsommer dans un sac de sable et donnée à la division [...]»; HIRSCHFELD, G., KRUMEICH, G., RENZ, I. Die Deutschen an der Somme 1914-1918, op. cit.

25. - Les dommages de guerre ne peuvent être dépensés que pour acquérir les biens qui ont fait l'objet de la demande d'indemnisation; la ville est donc "contrainte » de racheter des œuvres d'art pour le musée.

26. - Créée en 1926, l'association La Croûte regroupait artistes et amateurs d'art autour d'expositions régulières de peinture. Gelées en 1940, ses activités furent relancées au milieu des années 1970. L'association existe aujourd'hui, toujours très active, sous le nom de Société des peintres et amateurs d'art de Péronne et de sa région.

27. - Ensemble défensif du XVII ${ }^{\mathrm{e}}$ siècle qui protégeait le nord-est de Péronne, la Porte de Bretagne est très endommagée pendant la Grande Guerre. Elle est restaurée et classée Monument 
historique en 1923. Ses pavillons renferment de petites salles de quelques dizaines de mètres carrés chacune.

28. - Archives du musée Alfred-Danicourt, correspondance, 1938-1939.

29. - Gustave Devraine était vétérinaire de formation. Passionné de culture picarde et d'histoire, il fut désigné bibliothécaire et conservateur du musée péronnais à partir de 1940.

30. - Archives du musée Alfred-Danicourt, relation manuscrite par Gustave Devraine.

31. - Archives du musée Alfred-Danicourt, correspondance, 1941.

32. - Le Service Préhistoire et Archéologie, créé par les autorités allemandes, est notamment chargé de recenser les collections archéologiques françaises et de rapatrier en Allemagne les vestiges attribués à des peuples d'origine germanique. Voir BEN REDJEB, T. La carte archéologique de la Gaule, la Somme 80/2, p. 91, Académie des Inscriptions et Belles-Lettres, 2012.

33. - Fils du directeur du Service des travaux, Guy Savary fut témoin de l'affaire. Il raconte dans son ouvrage La vie municipale à Péronne que l'on répondit aux visiteurs allemands suivants que les objets avaient disparu.

34. - Archives du musée Alfred-Danicourt, correspondance, 1947.

35. - À cette époque, les reliquats du legs Danicourt de 1887 figurent encore aux recettes de la Ville.

36. - Archives du musée Alfred-Danicourt, correspondance, années 1950.

\section{ABSTRACTS}

The Alfred-Danicourt museum is located in the town hall of Péronne, a small sub-prefecture of the Somme department. Founded in 1877, and thus one of the oldest museums in Picardy, it was the creation of the wealthy collector Alfred Danicourt, who was also mayor of the town. The collection includes some exceptional archaeological ensembles, some antique cameos and some gallo-roman coins. When he died in 1887, Danicourt bequeathed his collection to the town along with an important sum of money for the upkeep of the museum. During the First World War the museum was destroyed by German troops and its collections plundered. But thanks to the courage of succeeding curators at the museum, a part of the collection was saved in 1914 and again in 1941. Through the dramas of twentieth-century history, the story of Danicourt's collections is a remarkable one.

Rêvé par un maire et un sous-préfet au XIXe siècle, le musée Alfred-Danicourt, musée municipal de la ville de Péronne depuis 1877, est toujours situé au cœur de l'hôtel de ville de cette petite sous-préfecture de la Somme. Il est l'un des plus anciens musées de Picardie. Certains de ses ensembles archéologiques font aujourd'hui référence, comme par exemple sa collection d'intailles et de camées antiques ou sa surprenante collection numismatique gauloise. La succession d'événements qui permit la préservation de ces trésors à travers l'histoire contemporaine est tout aussi exceptionnelle et doit beaucoup à l'initiative des conservateurs successifs de ce musée victime de guerre. Le destin des collections du musée Alfred-Danicourt est véritablement incroyable. 
INDEX

Mots-clés: Danicourt, musée, guerre mondiale, vol, pillage, protection, occupation, collections, Péronne

Keywords: Danicourt, museum, world war, theft, pillage, protection, military occupation, museum collections, Péronne

\section{AUTHOR}

DAVID DE SOUSA

Directeur du musée Alfred-Danicourt d.desousa@ville-peronne.fr 